\title{
Fine regulation of ARF17 for anther development and pollen formation
}

\author{
Bo Wang ${ }^{1,2}$, Jing-Shi Xue², Ya-Hui Yu², Si-Qi Liu², Jia-Xin Zhang ${ }^{2}$, Xiao-Zhen Yao², Zhi-Xue Liu*, \\ Xiao-Feng $\mathrm{Xu}^{2^{*}}$ and Zhong-Nan Yang ${ }^{2^{*}}$ (D)
}

\begin{abstract}
Background: In Arabidopsis, the tapetum and microsporocytes are critical for pollen formation. Previous studies have shown that ARF17 is expressed in microsporocytes and tetrads and directly regulates tetrad wall synthesis for pollen formation. ARF17 is the direct target of miR160, and promoterARF17:5mARF17 (5MARF17NT) transgenic plants, which have five silent mutations within the miR160-complementary domain, are sterile.

Results: Here, we found that ARF17 is also expressed in the tapetum, which was defective in arf17 mutants. Compared with arf17 mutants, 5 mARF17NT plants had abnormal tapetal cells and tetrads but were less vacuolated in the tapetum. Immunocytochemical assays showed that the ARF17 protein over-accumulated in tapetum, microsporocytes and tetrads of $5 \mathrm{mARF} 17 \mathrm{NT}$ plants at early anther stages, but its expression pattern was not affected during anther development. $5 \mathrm{mARF} 17$ driven by its native promoter did not rescue the arf17 male-sterile phenotype. The expression of 5 MARF17 driven by the tapetum-specific promoter A9 led to a defective tapetum and male sterility in transgenic plants. These results suggest that the overexpression of ARF17 in the tapetum and microsporocytes of 5 MARF17NT plants leads to male sterility. Microarray data revealed that an abundance of genes involved in transcription and translation are ectopically expressed in 5MARF17NT plants.
\end{abstract}

Conclusions: Our work shows that ARF17 plays an essential role in anther development and pollen formation, and ARF17 expression under miR160 regulation is critical for its function during anther development.

Keywords: Anther, Tapetum, Male sterility, ARF17, 5mARF17

\section{Background}

In flowering plants, male reproductive processes occur in the stamen. After meiosis, haploid microspores further develop into pollen grains within the locules. Each locule contains four somatic layers that surround microsporocytes/microspores/pollen [1]. The pollen wall is typically composed of an exine and intine. The innermost layer of locule sporophytic tissue, the tapetum, is in direct contact with the microspores to provide necessary nutrition and pollen wall materials for pollen grain maturation $[2,3]$. The deposition pattern of the exine is determined by callose formation during the tetrad stage $[4,5]$.

\footnotetext{
*Correspondence: liuzhixue@163.com; xiaofengxu@shnu.edu.cn; znyang@shnu.edu.cn

${ }^{1}$ Department of Molecular and Cell Biology, School of Life Science and Technology, Tongji University, Shanghai 200092, China

${ }^{2}$ College of Life and Environmental Sciences, Shanghai Normal University, Shanghai 200234, China
}

(c) The Author(s). 2017 Open Access This article is distributed under the terms of the Creative Commons Attribution 4.0 International License (http://creativecommons.org/licenses/by/4.0/, which permits unrestricted use, distribution, and reproduction in any medium, provided you give appropriate credit to the original author(s) and the source, provide a link to the Creative Commons license, and indicate if changes were made. The Creative Commons Public Domain Dedication waiver (http://creativecommons.org/publicdomain/zero/1.0/) applies to the data made available in this article, unless otherwise stated. ive development $[6,7]$. Both auxin biosynthesis and transportation are involved in pollen development [8-11]. Auxin response factors (ARFs) respond to auxin; ARFs can be targeted to auxin response elements of downstream genes and can function as transcriptional activators or repressors $[12,13]$. Several ARFs are involved in anther development and pollen formation. ARF1 and $A R F 2$ regulate flowering time and dehiscence [14], and $A R F 6$ and $A R F 8$ facilitate gynoecium and stamen development $[15,16]$. Loss of function of ARF17 directly affects the expression of CALLOSE SYNTHASE 5 (CALS5), which is critical for callose synthesis. arf 17 mutants show a thin callose wall and an abnormal exine pattern, resulting in male sterility $[4,17]$.

MicroRNAs (miRNAs) are 21 nucleotides long and are negative regulators of gene expression in both plants and animals [18]. Most plant miRNAs have the function of cleaving target mRNAs that have a perfect or nearly 
perfect complementary sequence [19]. In Arabidopsis, miR167 regulates ARF6 and ARF8 expression for ovule and anther development [15]. miR160 is complementary to the transcripts of ARF10, ARF16 and ARF17. Changing 5 bases of the miR160 recognition site without altering the amino acid sequence of the ARF17 protein (5mARF17) prevents miR160-directed ARF17 cleavage [20]. PromoterARF17::5mARF17 transgenic plants (5mARF17/WT) are sterile [20]. However, the details and mechanism of the effects of $5 m A R F 17$ on plant fertility are not clear.

Here, we show that ARF17 is important not only for microsporocyte/tetrad development but also for tapetum development. ARF17 protein was over-accumulated in the tapetum and microsporocytes, and this over-accumulation led to defects in $5 m A R F 17 / W T$ plants. Therefore, fine regulation of ARF17 is critical for tapetum development and pollen wall formation.

\section{Results}

ARF17 is essential for tapetum development

Previous investigations have shown that ARF17 directly regulates the expression of CALS5 for tetrad wall formation. In arf 17 mutants, tetrad walls are much thinner than those of wild type (WT), and the pollen wall pattern is defective [17]. In this work, we found that tapetum development was also defective in arf17 mutants and was abnormally vacuolated during development from stage 7 to stage 9 (Fig. 1a, b). Previous investigations have shown that the ARF17-GFP signal is localized in microsporocytes, microspores and mature pollen instead of tapetal cells [17]. Here, we rescanned ARF17-GFP signals using confocal laser scanning microscopy in pARF17::ARF17-GFP/arf17 complementary plants ( $\mathrm{T}_{3}$ generation) in which ARF17-GFP fully restored the arf17 male-sterile phenotype. The ARF17GFP signal was detected in tapetal cells at stage 5 , but then the signal weakened from stage 6 to 7 (Fig. 1c). The localization of ARF17-GFP in tapetal cells was consistent with the defective tapetum in the arf 17 mutants. These results suggest that ARF17 is also essential for tapetum development.

\section{$5 m A R F 17 / W T$ plants are defective in both the tapetum and microsporocytes}

ARF17 is among the miR160 targets in Arabidopsis, and promoterARF17::5mARF17 (5mARF17/WT) transgenic plants are sterile $[20,21]$. To better understand the role of ARF17 in male reproduction, we performed the same $5 m A R F 17$ construction and obtained 5mARF17/WT transgenic plants, which showed complete sterility as previously described (Additional file 1: Figure S1a-c; [20]). Alexander staining showed no mature pollen in
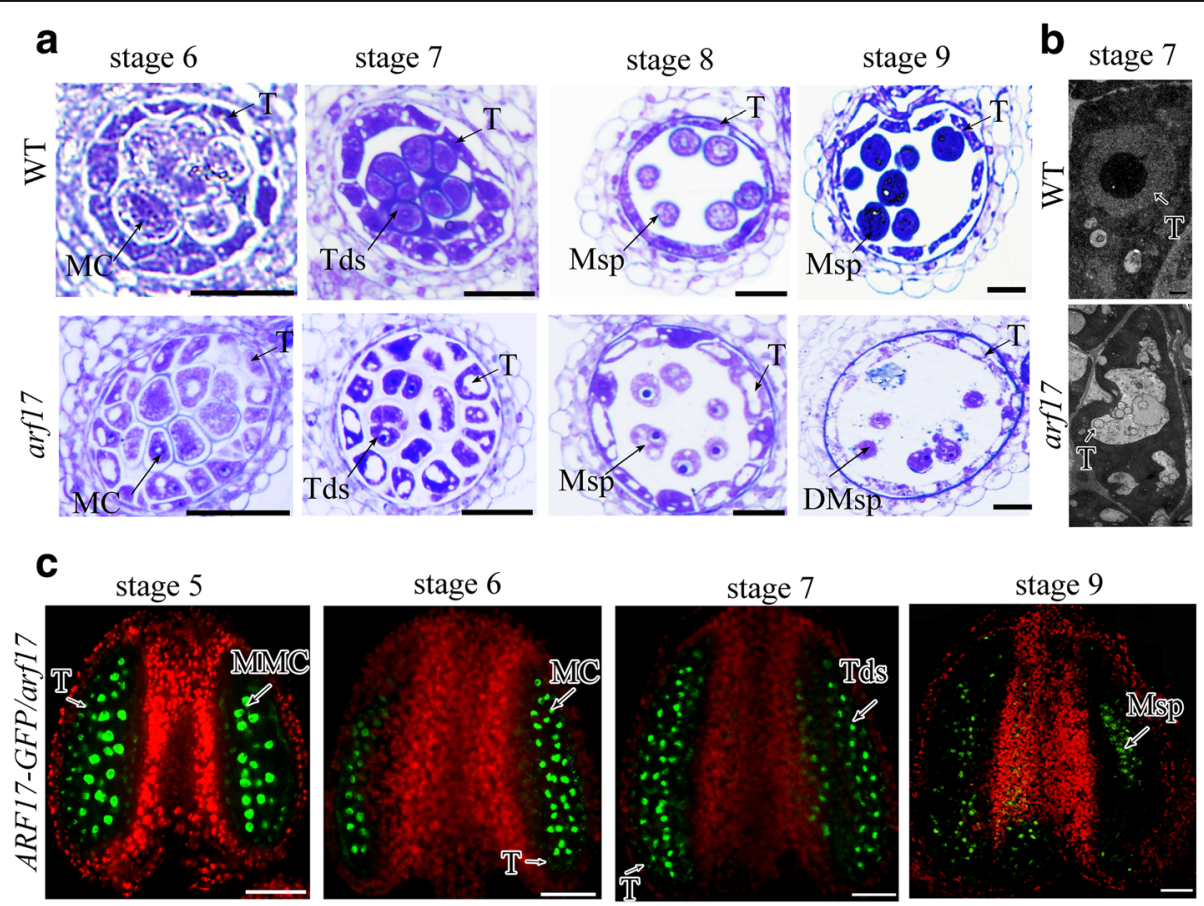

Fig. 1 ARF17 is important for tapetum development. a Semi-thin sections of the anthers of WT and arf17 mutant plants. Bar $=20 \mu \mathrm{m}$. b TEM showing the tapetum morphology of WT and arf17 plants at anther stage 7. Bar $=500 \mathrm{~nm}$. c Fluorescence confocal images indicating GFP signals in the anthers of an ARF17-GFP/arf17 plant. Bar $=50 \mu \mathrm{m}$. Green is the GFP signal $(530 \mathrm{~nm})$, and red is chlorophyll autofluorescence. DMsp, degenerated microspore; MC, meiotic cell; MMC, microspore mother cell; Msp, microspore; T, tapetum; Tds, tetrads 
the $5 m A R F 17$ WT plants, which was similar to observations in arf17 mutants (Fig. 2a-c). Reciprocal crosses with WT indicated that female fertility was not affected. Half of the $\mathrm{F}_{1}$ generation of $5 m A R F 17 \mathrm{NWT}$ plants pollinated with WT showed the male-sterile phenotype. PCR demonstrated that all these male-sterile plants contained the $5 m A R F 17$ fragment and that none of the fertile plants contained this fragment (Additional file 1: Figure S1d). These results suggest that $5 m A R F 17$ acts as a dominant gene for male sterility. Semi-thin sections showed no significant defects in anther development in the $5 m A R F 17 / W T$ plants before stage 6 . Tetrads formed normally in the $5 m A R F 17 / \mathrm{WT}$ plants at stage 7 (Fig. $2 \mathrm{~d}$ ), but the callose cell wall was much thinner than that in the WT plants (Additional file 1: Figure S1e-g). Tapetum development was also abnormal in the 5mARF17/ WT plants (Fig. 2d) but was less vacuolated than that in the arf17 mutants (Fig. 1a). At stage 8, individual microspores were observed in the $5 m A R F 17 / W T$ plants (Fig. 2d). Transmission electron microscopy (TEM) revealed that the exine of the $5 m A R F 17 / \mathrm{WT}$ plants was abnormal (Fig. 2e). After stage 8, the microspores became vacuolated and then degraded in the $5 m A R F 17$ plants (Fig. $2 \mathrm{~d})$. Therefore, both the tapetum and tetrad were defective in the $5 m A R F 17 / W T$ plants, which led to pollen rupture and male sterility.

\section{5mARF17 does not affect the expression of native ARF17}

The phenotype of the $5 m A R F 17 N$ WT plants regarding male development was similar to that of the arf17 mutants, except the tapetal cells (Fig. 1, Additional file 1: Figure S1a-c, Fig. 2d and e). To determine whether the $5 m A R F 17$ transgene affected the expression of the native $A R F 17,5 m A R F 17 / \mathrm{WT}$ plants were pollinated with pollen

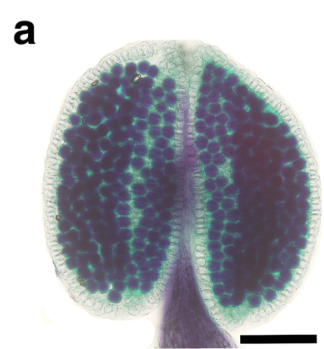

WT

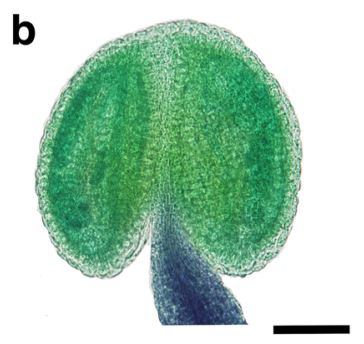

$5 m A R F 17 / \mathrm{WT}$

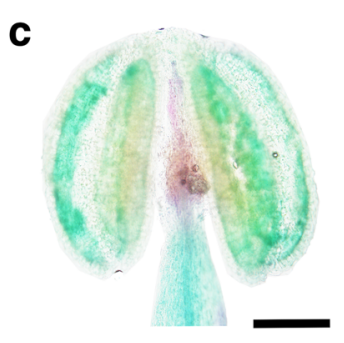

$\operatorname{arf17}$ d $\quad$ stage 6

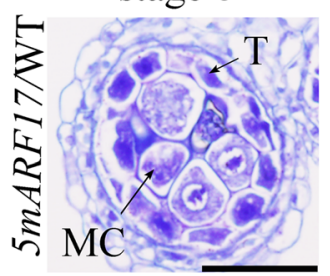

e

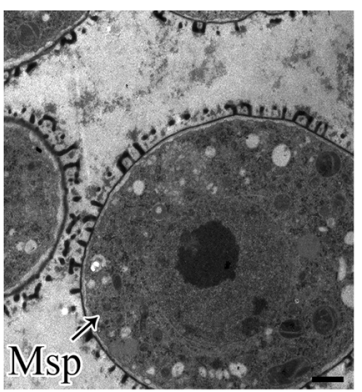

WT stage 7

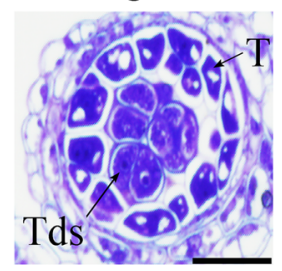

stage 9
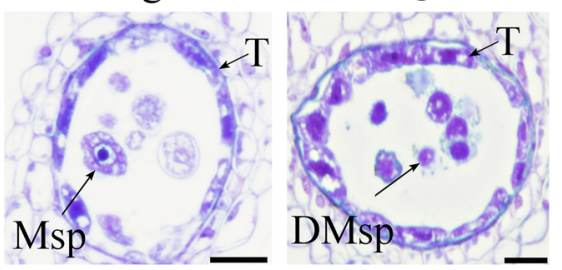

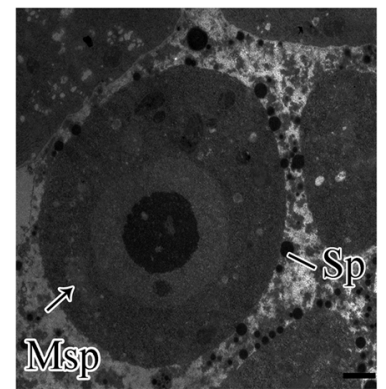

$5 m A R F 17 / \mathrm{WT}$

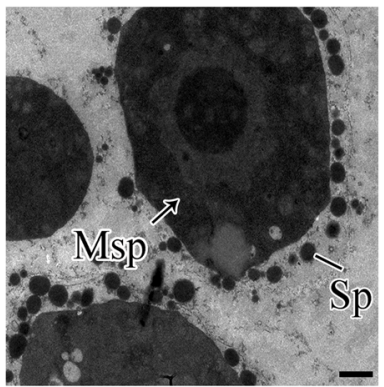

$\operatorname{arf17}$

Fig. 2 PromoterARF17::5mARF17 plants show complete male sterility. a-c Alexander's staining shows mature pollen in the anthers of a WT (a), a promoterARF17::5mARF17 (5mARF17NT) plant (b) and an arf17 mutant (c). Bars $=50 \mu \mathrm{m}$. (d) Semi-thin sections of the anthers of a $5 \mathrm{mARF} 17 \mathrm{WT}$ plant. Bar $=20 \mu \mathrm{m}$. (e) TEM showing the microspore structures of WT, $5 \mathrm{mARF} 17$ and arf17 mutant plants at anther stage 8. Bar $=5 \mu \mathrm{m}$. DMsp, degenerated microspore; MC, meiotic cell; Msp, microspore; T, tapetum; Tds, tetrads 
from ARF17-GFP/arf17 plants to obtain ARF17-GFP/ $5 m A R F 17$ plants. Because ARF17-GFP can complement the arf17 phenotype, the ARF17-GFP signal represents the expression of native ARF17. In the $5 m A R F 17 / W T$ plants, no GFP signals were observed (Fig. 3a). In the ARF17-GFP/5mARF17 plants, the ARF17-GFP signals were detected in microsporocytes, tetrads and microspores (Fig. 3b). The expression pattern of ARF17-GFP in the ARF17-GFP/5mARF17 plants was similar to that in the ARF17-GFP/arf17 plants (Fig. 1c), which represented the native expression. Thus, the $5 m A R F 17$ transgene did not affect the expression of the native ARF17.

\section{$5 m A R F 17$ cannot rescue the sterility of arf17}

The $5 m A R F 17$ transgene encodes the same amino acid sequence as does $A R F 17$ [20]. To investigate whether $5 m A R F 17$ fulfilled the same function as $A R F 17$, we crossed 5mARF17/WT plants with pollen from a heterozygous ARF17/arf17 plant. The $\mathrm{F}_{1}$ plants had the $5 m A R F 17$ transgene in the ARF17/arf17 background and were further crossed with an ARF17/arf17 plant to obtain a plant with the $5 m A R F 17$ transgene and an $\operatorname{arf} 17$ background (5mARF17/arf17) (Fig. 4a, Additional file 2: Figure S2a and b). We found that the 5mARF17/arf17 plants remained male sterile (Fig. $4 \mathrm{~b}$ ). The results of qRT-PCR showed that the expression level of ARF17 in the 5mARF17/arf17 plants was higher than that in the WT (Fig. 4c). Semi-thin sections of the anthers of the $5 m A R F 17 / a r f 17$ plants showed similar defects in the tapetum and microspores to those in the 5mARF17/WT plants (Figs. 2d and 4d). However, the tapetum showed less vacuolation than did the arf17 mutant (Fig. 1a). Therefore, the $5 m A R F 17$ transgene could partly rescue the tapetum development rather than recover male fertility in the arf 17 mutants. In our previous work, the same promoter of ARF17 used to construct $p A R$ F17::ARF17 complemented the arf17 mutant phenotype [17]. These results indicate that the escape of ARF17 expression from miR160 regulation leads to developmental defects in the anthers as well as male sterility.

ARF17 protein over-accumulates in the tapetum and microsporocytes of $5 \mathrm{mARF} 17 / \mathrm{WT}$ plants

In a previous study, miR160 failed to cleave $5 m A R F 17$ mRNA, and ARF17/5mARF17 mRNA over-accumulated in $5 m A R F 17 / W T$ plants [20]. To determine whether ARF17 protein also over-accumulated in the $5 m A R F 17 /$ WT plants, we constructed a promoterARF17::5mAR F17-GFP construct and then introduced it into a WT plant to obtain 5mARF17-GFP/WT transgenic plants. The 5mARF17-GFP/WT plants also showed a malesterile phenotype and a similar segregation ratio as that of the $5 m A R F 17 / W T$ plants (Additional file 3: Figure S3a-c). Confocal laser scanning microscopy was used to investigate GFP signals. During anther stages 5 to 7 , $5 \mathrm{mARF} 17-\mathrm{GFP}$ protein was expressed in the microsporocytes, microspores and tapetum. However, the GFP signals in the $5 m A R F 17-G F P / W T$ plants were much more diffuse than those of the ARF17-GFP plants
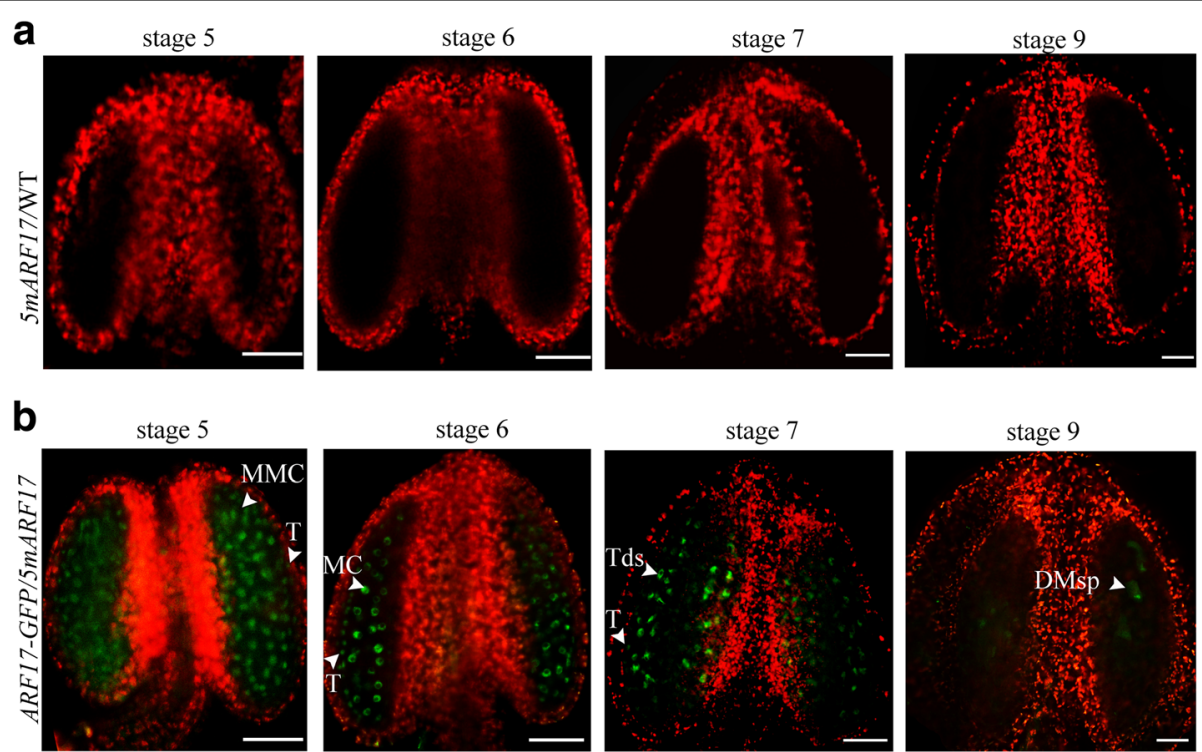

Fig. 3 Expression of native ARF17 was not affected in 5mARF17/NT plants. a and $\mathbf{b}$ Fluorescence confocal images indicating GFP signals in the anthers of a 5mARF17NT plant (a) and an ARF17-GFP/5mARF17 plant (b). Green is the GFP signal (530 nm), and red is chlorophyll autofluorescence. Bars = $50 \mu \mathrm{m}$. DMsp, degenerated microspore; MC, meiotic cell; MMC, microspore mother cell; T, tapetum; Tds, tetrads 

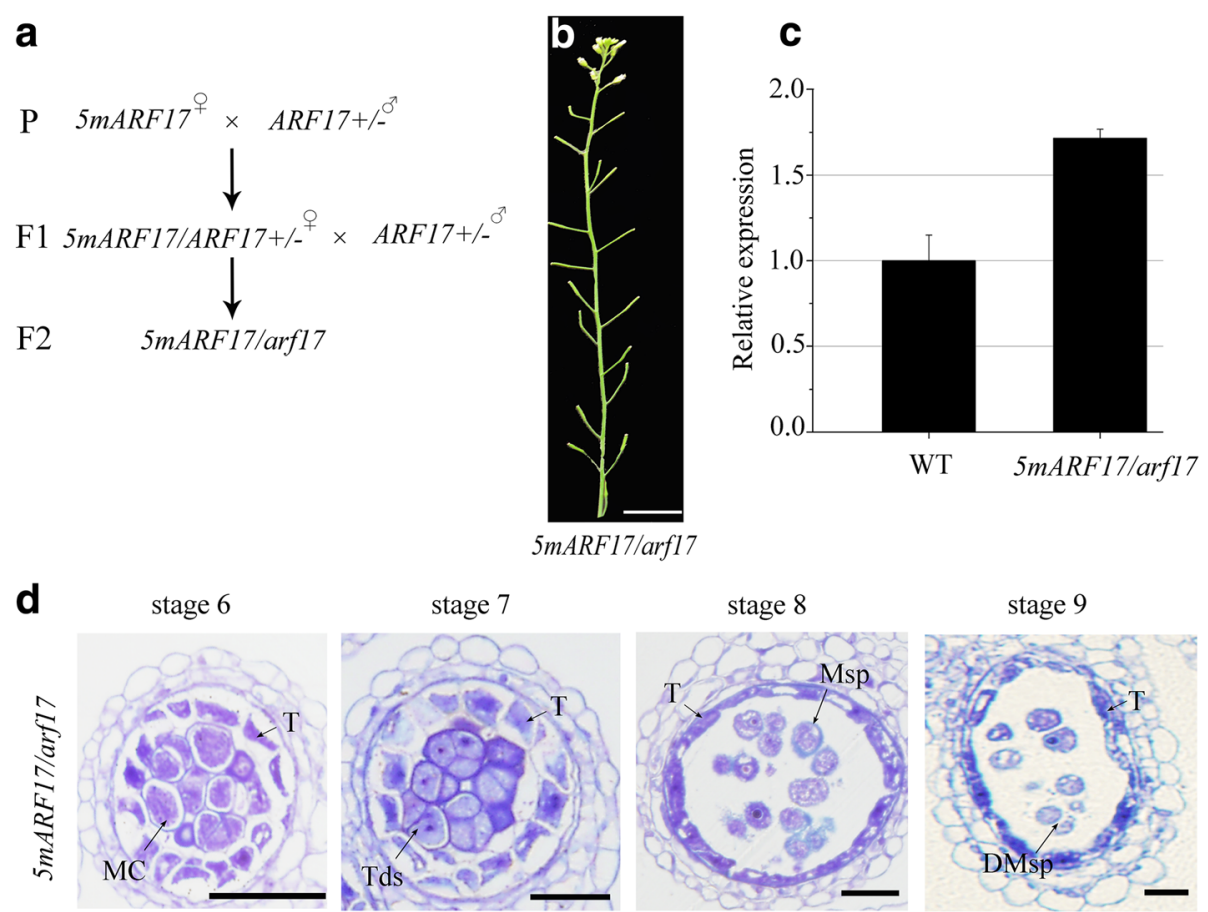

Fig. 4 Phenotype of $5 \mathrm{mARF} 17 / a r f 17$ mutants. a The schematic diagram indicates the steps to obtain a $5 \mathrm{mARF} 17 /$ arf17 plant. b A 35-day-old 5 mARF17/arf17 plant. Bar $=2 \mathrm{~cm}$. c Quantitative RT-PCR analysis of ARF17 expression in WT and 5mARF17/arf17 buds. The level of ARF17 was normalized to that tubulin and compared with that of WT. Error bars indicate SD and were calculated from three biological replicates. $\mathbf{d}$ Semithin sections of anthers in a 5mARF17/arf17 plant. Bar = $20 \mu \mathrm{m}$. DMsp, degenerated microspore; MC, meiotic cell; Msp, microspore; T, tapetum; Tds, tetrads

(Fig. 5a). Defects in the tapetum and microsporocytes could lead to the diffuse pattern of the ARF17-GFP signal in the 5mARF17-GFP/WT plants. To confirm whether ARF17 over-accumulated in the $5 \mathrm{mARF17/WT}$ plants, an immunohistochemical assay was employed using a GFP antibody to detect the accumulation of both 5mARF17-GFP in the 5mARF17NWT plants and ARF17-GFP in the ARF17-GFP/arf17 plants. In the ARF17-GFP/arf17 plants, the ARF17-GFP signal was observed in microsporocytes, tapetum, tetrads and microspores from stage 5 to stage 9 (Fig. $5 \mathrm{~b}$ ). In the 5mARF17-GFP/WT plants, 5mARF17-GFP proteins were more highly accumulated in the tapetum and $\mathrm{mi}$ crosporocytes than were the ARF17-GFP proteins in the ARF17-GFP/arf17 plants at stage 5. At stages 6-8, $5 \mathrm{mARF} 17-\mathrm{GFP}$ proteins continued to be expressed in the tapetum and microspores. Then, we harvested young buds (anther stage 5) from both $5 m A F R 17 / \mathrm{WT}$ and WT plants and investigated the expression of $A R F 17$ via qRT-PCR. The results of the qRT-PCR show that the expression of ARF17 in the $5 m A R F 17 / \mathrm{WT}$ plants is higher than that in the WT plants (Additional file 4: Figure S4b). These results indicated that 5 mARF17 protein over-accumulated in both the tapetum and microsporocytes at anther stage 5 in the $5 m A R F 17 / W T$ plants. This finding suggests $m i R 160$ is important for normal anther development in regulating ARF17 expression at an early anther stage.

Transgenic plants containing $5 m A R F 17$ driven by the tapetum-specific promoter $A 9$ are male sterile

Both the tapetum and microsporocytes contribute to pollen formation. To determine whether the overexpression of ARF17 in the tapetum leads to microspore abortion and male sterility, we constructed a promoterA9::5mARF17-GFP construct and introduced it into a WT plant. The $A 9$ promoter can drive gene expression specifically in the tapetum from anther stages 5 to 9 $[22,23]$. We found that the promoterA9::5mARF17GFP/WT transgenic plants ( $p A 9:: 5 m A R F 17-G F P / W T)$ were male sterile (Fig. 6a, b), and the segregation ratio was similar to that of $5 m A R F 17 / W T$ plants (Additional file 4: Figure S4a). The results of qRT-PCR showed that the expression of $A R F 17$ in the $p A 9:: 5 m A R F 17-G F P /$ WT plants was higher than that in the WT plants (Additional file 4: Figure S4c). The GFP signal in the $p A 9:: 5 m A R F 17-G F P / W T$ plants was restricted to the tapetum (Fig. 6c). Semi-thin sections showed the defects in tapetum development and pollen formation of the $p A 9:: 5 m A R F 17-G F P / W T$ plants; these defects were similar to those of $5 m A R F 17 / W T$ plants (Figs. $2 \mathrm{~d}$ and $6 \mathrm{~d})$. These results show that the over-accumulation of 

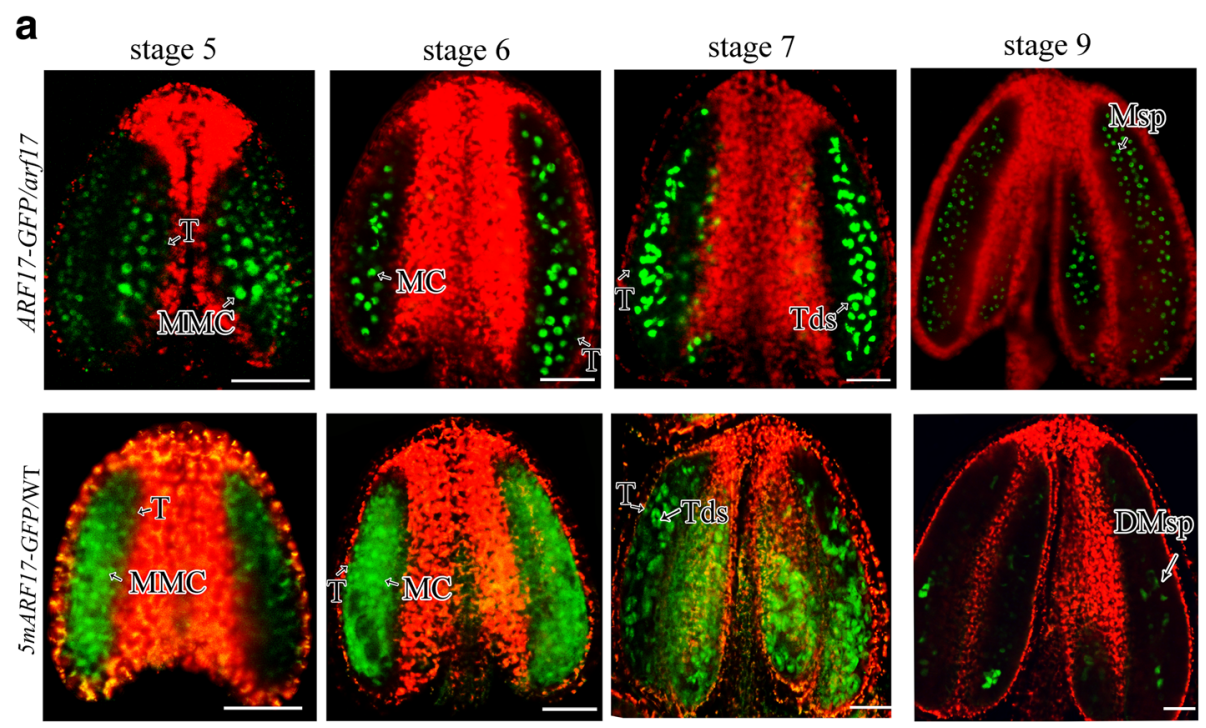

b

stage 5

stage 6

stage 7

stage 8

stage 9
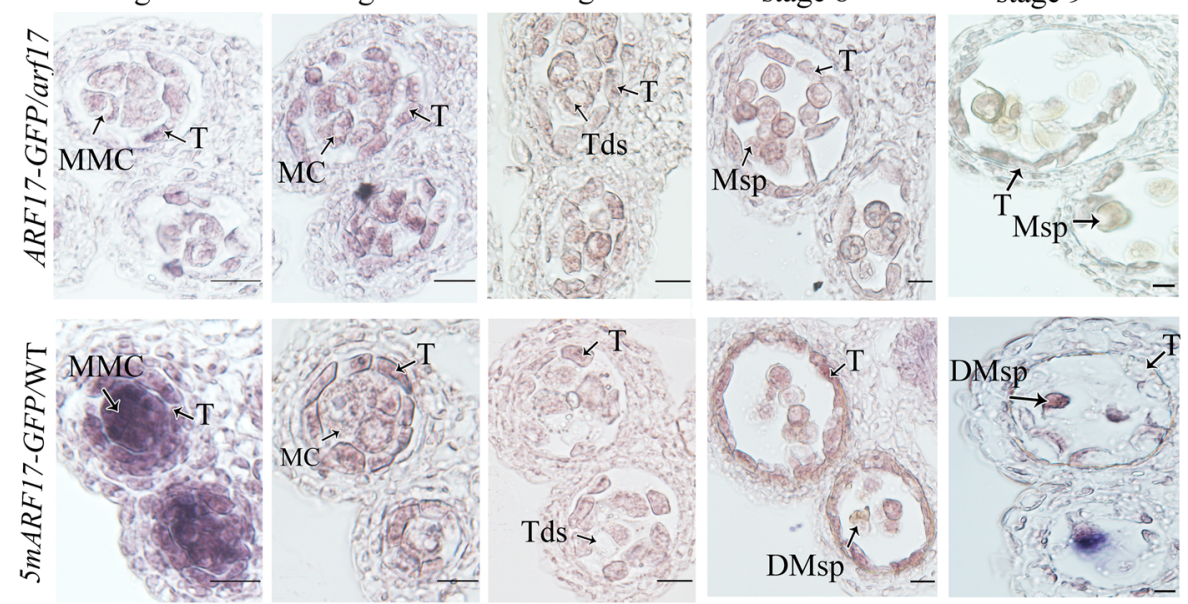

Fig. 5 GFP signals in ARF17-GFP/arf17 and 5mARF17-GFPNT anthers. a Fluorescence confocal images indicating GFP signals in the anthers of ARF17-GFP/ arf17 and 5mARF17-GFPNT plants. Bar $=50 \mu \mathrm{m}$. Green is the GFP signal $(530 \mathrm{~nm})$, and red is chlorophyll autofluorescence. $\mathbf{b}$ Immunohistochemical assays of GFP in the anthers at stages 5-10 of ARF17-GFP/arf17 and 5mARF17-GFPNT plants. Bar = $20 \mu \mathrm{m}$. DMsp, degenerated microspore; MC, meiotic cell; MMC, microspore mother cell; Msp, microspore; T, tapetum; Tds, tetrads

ARF17 in the tapetum was sufficient to lead to tapetum defects and plant sterility.

\section{Identification and overexpression of up-regulated genes in 5mARF17/WT plants}

Our previous work demonstrated that ARF17 functions as a transcriptional activator [17]. To identify the affected genes in $5 m A R F 17 / \mathrm{WT}$ plants, we performed a microarray analysis using young buds from the $5 m A R F 17 / \mathrm{WT}$ and WT plants. A total of 755 genes were up-regulated in $5 m A R F 17$ plants compared with WT plants (Additional file 5: Table S3). Based on the BAR information (http://bar.utoronto.ca), 220 (29.14\%) genes are ectopically expressed, and 339 (44.90\%) are overexpressed in $5 m A R F 17 / \mathrm{WT}$ plants (anther stage 5-7) (Additional file 5: Table S3). PANTHER (Protein Analysis Through Evolutionary Relationships, http://pantherdb.org) [24] analysis indicated that 316 known genes are overexpressed in $5 m A R F 17 N W T$ buds (Fig. 6a; Additional file 6: Table S4), with 77 of the 316 genes associated with nucleic acid binding (24.4\%). In this group, DNA helicase, RNA binding protein, ribosomal protein and histones were highly enriched (Additional file 6: Table S4). These results indicate that overexpression of ARF17 may lead to alterations of normal gene expression, resulting in disruption of anther and tapetum development.

To test whether the expression of some of the upregulated genes in the $5 m A R F 17 / \mathrm{WT}$ plants affect anther development and pollen formation, we fused some of the genes to CaMV35S or A9 promoters, and the 


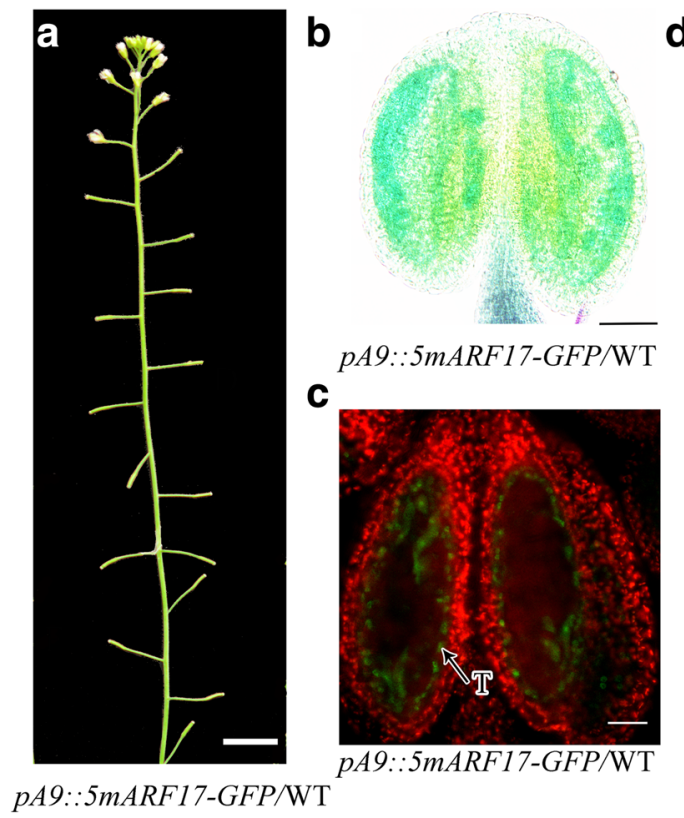

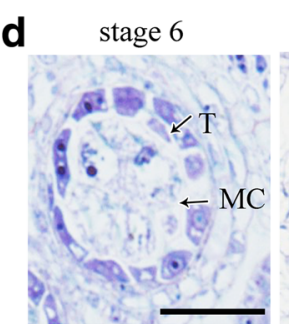

stage 8

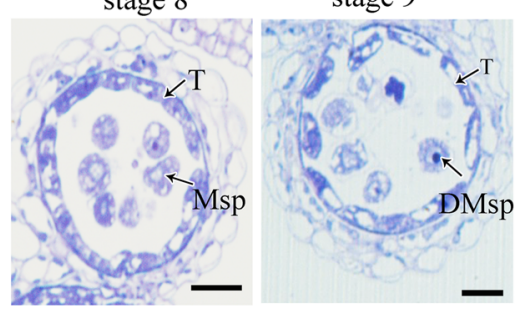

pA9::5mARF17-GFP/WT stage 7

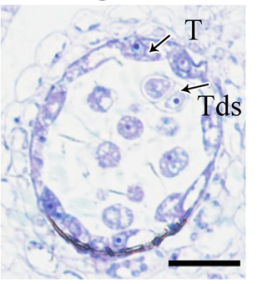

stage 9

Fig. 6 Phenotype of a promoterA9::5mARF17-GFPMT plant. a A 35-day-old promoterA9::5mARF17-GFP (pA9::5mARF17-GFPMT) plant. Bar = 2 cm. b Alexander's staining of the anthers of a $p A 9:: 5 m A R F 17-G F P / W T$ plant. Bar $=200 \mu \mathrm{m}$. c GFP signals were observed exclusively in the tapetal cells of pA9::5mARF17-GFP/WT plants. Bar $=50 \mu \mathrm{m}$. d Semi-thin sections of the anthers of a pA9::5mARF17-GFP/WT plant showing pollen development from anther stages 6-9. Bar = 20 Mm. DMsp, degenerated microspore; MC, meiotic cell; Msp, microspore; T, tapetum; Tds, tetrads

resulting constructs were introduced into WT plants. AT1G48640 encodes a transmembrane amino acid transporter family gene that is involved in root development [25]. 12/12 transgenic lines with over-expression of AT1G48640 were fertile in promoterA9::AT1G48640/WT transgenic plants (Additional file 7: Figure S6B, Fig. 7b and c), but their pollen grains adhered together (Fig. 7d, e), and scanning electron microscopy (SEM) showed defects in pollen wall structure in those plants (Fig. 7j, k). Zinc-finger protein 1 (ZF1) functions as a transcriptional repressor and is involved in the inhibition of plant growth under abiotic stress conditions [25-27]. 8/8 transgenic lines with over-expression of $Z F 1$ showed reduced fertility in CaMV35s::ZF1/WT plants (Additional file 7: Figure S6b, Fig. $7 \mathrm{f}$ and $\mathrm{g}$ ). Some pollen was aborted and adhered together in the CaMV35s::ZF1/WT plants (Fig. 7h, i), and SEM showed that pollen wall formation was also defective in these plants (Fig. 7j, l).

\section{Discussion}

ARF17 is important for both tapetum and microsporocyte development

ARF17 is a member of the ARF family in Arabidopsis. In arf17 mutants, all pollen is ruptured, and plants show male sterility [17]. During anther development, the microsporocyte/tetrad determines the pollen wall pattern, whereas tapetal cells provide both nutrition and pollen wall materials for pollen formation. ARF17 is expressed in microsporocytes and tetrads during anther development and directly regulates the expression of CALS5 for both tetrad wall synthesis and exine pattern formation [17]. In this work, ARF17 expression was also detected in the tapetum based on the ARF17-GFP signal and immunocytochemical assay results (Figs. 1c and 5b). The ARF17-GFP signals were detected in the tapetum at an early stage for a short period. In addition, the signal was much weaker than that in the microsporocytes/tetrads (Fig. 1c), with the weaker signal the primary reason that the expression was not detected in the tapetum in previous studies [17]. However, the short expression period was important for the development of the tapetum, with the tapetum clearly defective in the arf17 mutants (Fig. 1a). After stage 4, a key genetic pathway of DYT1-TDF1-AMS is important for tapetum development and pollen wall formation [28-31]. DYT1 is the earliest transcription factor required for tapetum development and is initially detected at approximately stage 5 [28]. In the arf17 mutants, DYT1, TDF1, and $A M S$ expression was not significantly affected (Additional file 8: Figure S5a), and in $d y t 1$ and $t d f 1 \mathrm{mu}-$ tants, ARF17 transcription was not dramatically affected (Additional file 8: Figure S5b). Therefore, ARF17 plays a critical role in early tapetum development and may be independent of the DYT1-TDF1-AMS pathway.

\section{Overexpression of ARF17 in 5mARF17/WT plants leads to defective microsporocytes and tapetum}

Previous studies have shown that $5 m A R F 17 / \mathrm{WT}$ plants are sterile [20]. Reciprocal crosses with wild-type plants 
a $5 m A R F 17 /$ WT VS WT (up-regulated) Panther Go-Slim Protein Class

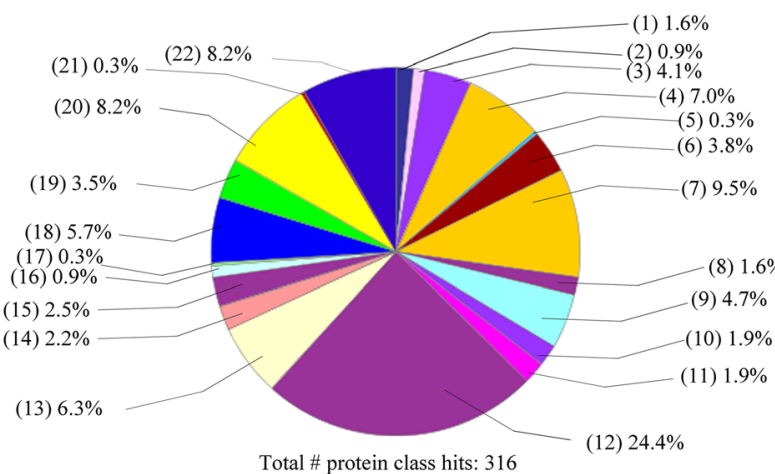

b

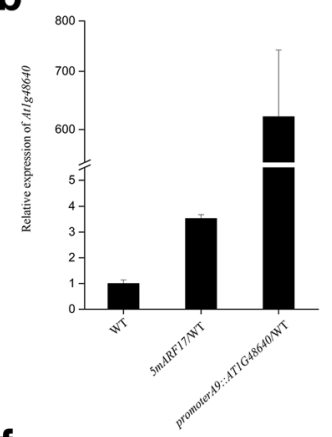

f

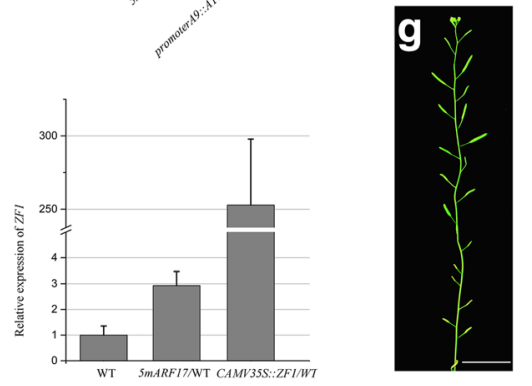

d
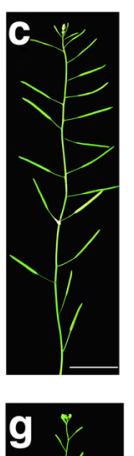

g
(1) calcium-binding protein (2) cell adhesion molecule (3) chaperone

(4) cytoskeletal protein

(5) defense/immunity protein

(6) enzyme modulator

(7) hydrolase

(8) isomerase

(9) ligase

(10) lyase

(11) membrane traffic protein

(12) nucleic acid binding

(13) oxidoreductase

(14) receptor

(15) signaling molecule

(16) storage protein

(17) structural protein

- (18) transcription factor

(19) transfer/carrier

(20) transferase

(21) transmembran

(22) transporter

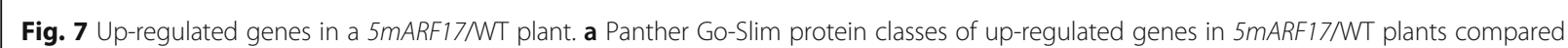
with those in WT. The total hits included 316 genes. The percentage of each term is the clustered gene number of the total protein class hits: calcium binding (5 genes), cell adhesion molecule (3 genes), chaperone (13 genes), cytoskeletal protein (22 genes), defense immunity protein (1 gene), enzyme modulator (12 genes), hydrolase (30 genes), isomerase (5 genes), ligase (15 genes), lyase (6 genes), membrane traffic protein (6 genes), nucleic acid binding (77 genes), oxidoreductase (20 genes), receptor (7 genes), signaling molecule (8 genes), storage protein (3 genes), structural protein (1 gene), transcription factor (18 genes), transfer/carrier protein (11 genes), transferase (26 genes), transmembrane receptor regulatory/adaptor protein (1 gene), and transporter (26 genes). b-I The phenotypes of promoterA9::AT1G48640 and CAMV35s::ZF1 plants. b Quantitative RT-PCR analysis of AT1G48640 expression in WT, 5mARF17NT and promoterA9::AT1G48640NT buds. c A 35-day-old promoterA9:AT1G48640/ WT plant. Bar $=2 \mathrm{~cm}$. $\mathbf{d}$ and $\mathbf{e}$ Alexander's staining of the anthers and pollen grains of a promoterA9:AT1G48640NT plant. Bars $=20 \mu \mathrm{m}$. $\mathbf{f}$ Quantitative RT-PCR analysis of ZF1 expression in WT, 5mARF17NT and CAMV355.:ZF1MT buds. g A 35-day-old CAMV35s.:ZF1MT plant. Bar $=2 \mathrm{~cm}$. $\mathbf{h}$ and $\mathbf{i}$ Alexander's staining of the anthers and pollen grains of a CAMV355::ZF1MT plant. Bars $=20 \mu \mathrm{m}$. $\mathbf{j}-\mathbf{I}$ Scanning electron microscopy (SEM) showing the pollen wall structures of a WT plant (j), a promoterA9::AT1G48640NT plant (k) and a CAMV35s::ZF1NT plant (I). Bars = 5 um. The levels of AT1G48640 and ZF1 were normalized to those of tubulin and compared with those of WT. Error bars indicate SD and were calculated from three biological replicates

showed that the female parts are not affected in the $5 m A R F 17 / \mathrm{WT}$ plants (Additional file 1: Figure S1d). The $5 m A R F 17 / N T$ plants had no mature pollen inside anther locules, and both the tapetum and microsporocytes/ tetrads were defective (Fig. 2a-e). Overall, the cellular defects of anther development were similar between the arf17 and 5mARF17/WT plants. However, the defective tapetum development in the arf 17 mutants is more severe than that in the $5 m A R F 17 / \mathrm{WT}$ plants after stage 6 (Additional file 7: Figure S6a). In the arf 17 mutants, the loss of ARF17 function led to pollen rupture and male sterility. In $5 m A R F 17 / \mathrm{WT}$ plants, the transgene $5 m A R F 17$ did not affect the expression of the native ARF17 (Fig. 3a and b). Although 5mARF17 encodes the same protein as does $A R F 17$, it could not complement the male sterility of arf17 mutants (Fig. 4b). Hence, the 
mechanisms that lead to the male sterility of both $5 m A R F 17 / W T$ and arf17 plants are different. In the $5 m A R F 17 /$ WT plants, ARF17 protein over-accumulated in the tapetum and microsporocytes (Fig. 5a, b). The tapetum-specific over-accumulation of ARF17 was sufficient to cause male sterility, which was similar to that observed for the 5mARF17/WT plants (Fig. 6a-d). Therefore, the overexpression of ARF17 in the tapetum of the $5 m A R F 17 / \mathrm{WT}$ plants prevents normal tapetum function. The microsporocytes/tetrads are also defective in the $5 m A R F 17 /$ WT plants. It is likely that the overexpression of ARF17 in the microsporocytes/tetrads of the $5 m A R F 17 / \mathrm{WT}$ plants leads to its male sterility. ARF17 directly regulates the expression of CALS5, which is needed for tetrad wall formation. In the arf 17 mutants, the expression of CALS5 is significantly reduced [17]. The 5mARF17/WT plants also show defective tetrad walls and reduced expression of CALS5 (Additional file 1: Figure S1e-h). It is likely that these phenomena are a side effect of defective microsporocyte/tetrad development in the $5 m A R F 17 / \mathrm{WT}$ plants. The results of the microarray analysis showed that $5 m A R F 17$ activates the ectopic expression of many genes in the anthers of the $5 m A R F 17 / W T$ plants. Overexpression of two of these genes, AT1G48640 and ZF1, slightly affected pollen formation and plant fertility (Fig. $7 \mathrm{~b}-\mathrm{l}$ ). Therefore, the male sterility of the $5 m A R F 17 / \mathrm{NT}$ plants resulted from the ectopic expression and overexpression of these genes, including AT1G48640 and ZF1.

\section{Expression of ARF17 is precisely regulated during anther development}

ARF17 is among the targets of miR160 [20,32], and the knockout of $A R F 17$ leads to male sterility [17]. In this work, we demonstrated that without miR160 control in $5 m A R F 17 / W T$ plants, ARF17 was overexpressed, which led to male sterility. Therefore, the expression level of ARF17 is critical for pollen development. The expression pattern of 5mARF17-GFP was apparently similar to that of ARF17-GFP (Fig. 5b). Therefore, the ARF17 promoter determined the cell and tissue specificity of ARF17 expression, whereas miR160 controlled the level of ARF17 expression. In Arabidopsis, three precursors, including miR160a, miR160b and miR160c, can produce miR160 [20]. In humans, the TRISTETRAPROLINE (TTP) protein is a member of the RNA-induced silencing complex (RISC) [33, 34]. AtTTP is an ortholog of hTTP in Arabidopsis and is expressed in microsporocytes, tetrads and tapetal cells, and AtTTP expression highly overlapped with that of ARF17. Overexpression of AtTTP decreases the level of mature miR160, whereas the expression of ARF17 increases it [35]. Thus, AtTTP is involved in miR160 maturation for the fine regulation of ARF17 for pollen formation during anther development.

\section{Conclusion}

ARF17 is the target of miR160, and 5mARF17/WT plants show male sterility as do arf17 plants. In this study, we showed that ARF17 plays an essential role in anther development and pollen formation. Without miR160 regulation, the expression pattern of $A R F 17$ in the anthers is not affected, but its expression level is significantly elevated in $5 m A R F 17 / \mathrm{WT}$ plants. The overexpression of ARF17 in $5 m A R F 17$ plants leads to defects in pollen formation and plant sterility.

\section{Methods}

\section{Plant materials and growth conditions}

In this study, Arabidopsis (Arabidopsis thaliana) wildtype (WT), transgenic and mutant plants in the Col-0 ecotype background were used. arf17 mutants and $5 m A R F 17 / W T$ plants were preserved in the laboratory of Z.N. Yang. Seeds were sown on vermiculite and allowed to imbibe for 2 to 3 days. Plants were grown under conditions of $16 \mathrm{~h}$ light $/ 8 \mathrm{~h}$ darkness in a growth chamber for approximately 22 days.

\section{Microscopy}

The plants were imaged with a Cyber Shot T-20 digital camera (Sony). Alexander staining solution was prepared as described [23]. The anthers were stained for approximately $2-12 \mathrm{~h}$ at room temperature (RT, $22{ }^{\circ} \mathrm{C}$ ). For semi-thin sections, the flower buds were fixed and embedded in Spurr's resin. Semi-thin sections were prepared by cutting the buds to a thickness of $1 \mu \mathrm{m}$ followed by incubation in a $0.01 \%$ toluidine blue/sodium borate solution for $5-10 \mathrm{~min}$ at $42{ }^{\circ} \mathrm{C}$, after which the sections were washed with water. The sections were observed with an Olympus BX51 microscope under brightfield microscopy.

\section{Fluorescence microscopy}

For callose staining, anthers at the tetrad stage were squeezed onto a slide and stained with toluidine blue as described previously [17]. GFP signals in the anthers of ARF17-GFP/arf17, 5mARF17-GFP/WT, and ARF17GFP/5mARF17 plants were observed using a Carl Zeiss confocal laser scanning microscope (LSM 5 PASCAL).

\section{Electron microscopy}

For TEM examination, flower buds were fixed in $0.1 \mathrm{M}$ phosphate buffer ( $\mathrm{pH} 7.2)$ with $2.5 \%$ glutaraldehyde $(\mathrm{v} / \mathrm{v})$ and then washed several times with PBS ( $\mathrm{pH} 7.4)$, followed by dehydration with ethyl alcohol and replacement by propylene epoxide. The samples were embedded in Spurr's resin and polymerized for $48 \mathrm{~h}$ at $60{ }^{\circ} \mathrm{C}$ as described previously [23, 30]. Then, TEM microscopy (JEOL, Japan) was used to observe the slides. For SEM observations, fresh pollen grains were coated with $8 \mathrm{~nm}$ 
of gold and observed under a JSM-840 microscope (JEOL) [23].

Plasmid construction and identification of transgenic plants The promoterARF17::5mARF17 genomic region was cloned using ARF17 primers (ProARF17-F/R) and (5mARF17g-F/ $\mathrm{R})$ to complement the wild type (WT) in accordance with Mallory identification [20]. The promoterA9::5mARF17GFP fragment was constructed with the $A 9$ promoter (ProA9-F/R) and the $5 m A R F 17$ genomic region to complement the WT. For GFP fusion, the $5 m A R F 17$ genomic fragment without a stop codon was cloned into a modified GFP-pCAMBIA1300 vector using primers (5mARF17g-F/ 5mARF17g-GFP-R) to complement the WT. The promoterA9::AT1G48640 fragment was constructed with the $A 9$ promoter (ProA9-F/R) and the AT1G48640 CDS (4864-F/ 4864-R) region transferred into the WT. The CaMV35:ZF1 fragment was constructed with the CAMV35 promoter (35 s-F/35 s-R) and the ZF1 CDS (ZF1-F/ZF1-R) region introduced into the WT. These fragments were amplified using KOD polymerase (Takara Biotechnology) and cloned into the pCAMBIA1300 and GFP-pCAMBIA1300 vectors (CAMBIA). The fragments were verified by sequencing. The plasmids were transformed into Agrobacterium tumefaciens (GV3101) and screened using $50 \mathrm{mg} / \mathrm{ml}$ kanamycin, $40 \mathrm{mg} / \mathrm{ml}$ gentamicin and $50 \mathrm{mg} / \mathrm{ml}$ rifampicin. The bacteria containing the plasmid constructs were introduced into the flower buds. The transgenic plants were selected using $20 \mathrm{mg} / \mathrm{l}$ hygromycin. The primer sequences are listed in Additional file 9: Table S1.

\section{Immunohistochemical staining}

For immunohistochemical staining, flower buds were fixed in formaldehyde/acetic acid for 1 day, dehydrated in an ethanol gradient and embedded in wax. Sections that were $8 \mathrm{~mm}$ thick were prepared using a rotary microtome (MR2; RMC). The sections were incubated in boiled citrate buffer ( $\mathrm{pH}$ 6.4) for 10-15 min after rehydration and then cooled to RT. The slides were washed twice in PBS ( $\mathrm{pH}$ 7.4) for 5 min. Then, the sections were blocked with $5 \%$ BSA in PBS for $30 \mathrm{~min}$ to $1 \mathrm{~h}$. Rabbit anti- $6 \times$ GFP antibodies (Thermo Scientific) were diluted 1:100 in PBS (pH 7.4), and the slides were then incubated at $4{ }^{\circ} \mathrm{C}$ overnight. Then, the slides were washed three times in Tris-buffered saline solution (TBS; pH 7.4) for 5 min and incubated with anti-rabbitAP antibodies diluted 1:200 in TBS (pH 7.4) for $1 \mathrm{~h}$ at RT. The slides were washed three times in TBS for 5 min, and BCIP/NBT solution (CWBIO) was used for colorimetric detection at RT.

\section{Quantitative RT-PCR}

Total RNA was extracted from the flower buds of the WT, transgenic and mutant plants using a TRIzol kit
(Invitrogen). In accordance with the manufacturer's instructions (Toyobo), first-strand complementary DNA (cDNA) was synthesized. Quantitative RT-PCR was performed using an ABI PRISM 7300 detection system (Applied Biosystems) with SYBR Green I master mix (Toyobo). The primers ARF17qRT-F/R were used to detect the expression level of ARF17. The relative expression levels were calculated according to the cycle numbers. The qRT-PCR results are shown as the relative expression levels normalized to those of tubulin. The positive control was the tubulin gene (TUB-F/R), and three replicates were performed for each experiment. The relevant primer sequences are listed in Additional file 10: Table S2.

\section{Microarrays}

Microarrays were performed according to a previously described procedure [30]. Young buds collected from WT and $5 m A R F 17 /$ WT plants were immediately frozen in liquid nitrogen. A Low-RNA-Input Linear Amplification Kit (Agilent Technologies) was used to amplify and label the total RNA. 5-(3-Aminoallyl)-UTP (Ambion), Cy3 NHS ester (GE Healthcare Biosciences) and Cy5 NHS ester (GE Healthcare Biosciences) were applied following the manufacturers' instructions. The labeled cRNA was purified using an RNeasy Mini Kit (Qiagen). According to the manufacturer's instructions, each 44-K Arabidopsis oligo microarray slide was hybridized with $825 \mathrm{ng}$ of Cy3-labeled cRNA and $825 \mathrm{ng}$ of Cy5-labeled cRNA using a gene expression hybridization kit (Agilent) in a hybridization oven (Agilent). The slides were scanned using an Agilent Microarray Scanner (Agilent) and Feature Extraction software 10.7 (Agilent) with the default settings. Three biological replicates of independently grown materials were used. The raw data were normalized with a locally weighted scatter plot smoothing (Lowess) algorithm using Gene Spring Software 11.0 (Agilent).

\section{Additional files}

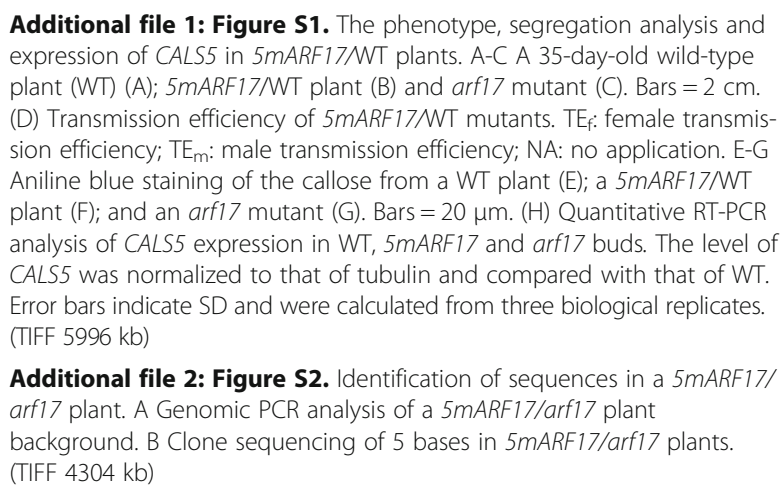

Additional file 1: Figure S1. The phenotype, segregation analysis and

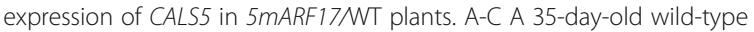
plant (WT) (A); $5 m A R F 17 / \mathrm{WT}$ plant (B) and $\operatorname{arf17}$ mutant (C). Bars $=2 \mathrm{~cm}$. (D) Transmission efficiency of 5 MARF17NT mutants. TE. female transmission efficiency; $T E_{m}$ : male transmission efficiency; NA: no application. E-G Aniline blue staining of the callose from a WT plant (E); a 5 MARF17/WT plant (F); and an arf17 mutant (G). Bars $=20 \mu \mathrm{m}$. (H) Quantitative RT-PCR analysis of CALS5 expression in WT, 5 MARF17 and arf17 buds. The level of CALS5 was normalized to that of tubulin and compared with that of WT. Error bars indicate SD and were calculated from three biological replicates. (TIFF 5996 kb)

Additional file 2: Figure S2. Identification of sequences in a $5 \mathrm{mARF17/}$ arf17 plant. A Genomic PCR analysis of a 5 mARF17/arf17 plant background. B Clone sequencing of 5 bases in 5 mARF17/arf17 plants. (TIFF 4304 kb) 
Additional file 3: Figure S3. Phenotype and segregation analyses of 5 MARF17-GFPNT plants. A and B A 35-day-old 5mARF17-GFPNT plant (A) and ARF17-GFP/5mARF17 plant (B). Bars $=2 \mathrm{~cm}$. (C) Transmission efficiency of a 5 mARF17-GFPNT plant. TE: female transmission efficiency; $T E_{m}$ : male transmission efficiency; NA: no application. (TIFF $1132 \mathrm{~kb}$ )

Additional file 4: Figure S4. Transmission efficiency of a $p A 9:: 5 m A R F 17-$ GFP/WT plant. A Transmission efficiency of a promoterA9::5mARF17-GFP/ WT (pA9::5mARF17-GFPNT) plant. B Quantitative RT-PCR analysis of ARF17 expression in WT and 5mARF17/WT buds at stage 5. C Quantitative RTPCR analysis of ARF17 expression in WT and pA9::5mARF17-GFPNT buds. The level of ARF17 was normalized to that of tubulin and compared with that of WT. Error bars indicate SD and were calculated from three biological replicates. $\mathrm{TE}_{\mathrm{F}}$ : female transmission efficiency; $\mathrm{TE}_{\mathrm{m}}$ : male transmission efficiency; NA: no application. (TIFF $491 \mathrm{~kb}$ )

Additional file 5: Table S3. List of up-regulated genes in 5mARF17/WT that are typically expressed or absent in WT buds. (XLSX $65 \mathrm{~kb}$ )

Additional file 6: Table S4. Protein classification of up-regulated genes by PANTHER. (XLS $116 \mathrm{~kb}$ )

Additional file 7: Figure S6. Semi-thin sections of anthers and PCR analysis. A Semi-thin sections of anthers in WT, arf17 and 5mARF17/NT plants from stage 5 to 9 . Bar $=20 \mu \mathrm{m}$. B Identification of transgenic sequences in WT, promoterA9:: AT1G48640/WT and CAMV355::ZF1/WT. DMsp, degenerated microspore; MC, meiotic cell; MMC, microspore mother cell; Msp, microspore; T, tapetum; Tds, tetrads. (TIFF $23380 \mathrm{~kb}$ )

Additional file 8: Figure S5. Relative expression of tapetum- and pollen-formation genes in 5mARF17/NT and arf17 plants. A Quantitative RT-PCR analysis of the expression levels of genes involved in tapetum and pollen development. B Quantitative RT-PCR analysis of ARF17 expression in dyt1 and tdf1 mutants. The levels of DYT1, TDF1, TEK, CDKG1, RPG1, MS188, MS1, ACOS5, and ARF17 were normalized to those of tubulin and compared with those of WT. Error bars indicate SD and were calculated from three biological replicates. (TIFF $937 \mathrm{~kb}$ )

Additional file 9: Table S1. List of primers used in clones and identification. (XLS $21 \mathrm{~kb}$ )

Additional file 10: Table S2 List of primers used in qRT-PCR. (XLS $22 \mathrm{~kb})$

\section{Acknowledgments}

We thank Jun Yang for his help with the construction of promoterARF17::5mARF17 plants.

\section{Funding}

This work was supported by grants from the Major Research Plan from the Ministry of Science and Technology of China (2013CB945100), the National Science Foundation of China (31670314) and the National Science Foundation of China (31401030). The funders had no role in study design, data collection and analysis, decision to publish, or preparation of the manuscript.

\section{Availability of data and materials}

The data supporting the results of this article are included within the article and its additional files. The gene and promoter sequences of ARF17 were deposited in TAIR (https://www.arabidopsis.org) under accession number AT1G77850.

\section{Authors' contributions}

$Z X L$ and $Z N Y$ administered the project; $X X F, Z X L$ and $Z N Y$ conceived the original screening and research plans; ZNY supervised the experiments; BW performed most of the experiments; YHY, SQL and JXZ were involved in performing the experiments; BW and XXF designed the experiments, analyzed the data and wrote the article with contributions from JSX and XZY; and ZNY reviewed and edited the writing. All authors read and approved the final manuscript.

\section{Ethics approval and consent to participate}

Not applicable.

\section{Consent for publication}

Not applicable.

\section{Competing interests}

The authors declare that they have no competing interests.

\section{Publisher's Note}

Springer Nature remains neutral with regard to jurisdictional claims in published maps and institutional affiliations.

Received: 27 July 2017 Accepted: 27 November 2017

Published online: 19 December 2017

\section{References}

1. Goldberg RB, Beals TP, Sanders PM. Anther development: basic principles and practical applications. Plant Cell. 1993;5(10):1217-29.

2. Heslop-Harrison PJ. Origin of Exine. Nature. 1962;195(4846):1069-71.

3. Mariani C, Beuckeleer, M.D., Truettner, J., Leemans, J., and Goldberg, R.B.: Induction of male sterility in plants by a chimaeric ribonuclease gene. Nature 1990, 347(6295):737-741.

4. Dong X, Hong Z, Sivaramakrishnan M, Mahfouz M, Verma DP. Callose synthase (CalS5) is required for exine formation during microgametogenesis and for pollen viability in Arabidopsis. Plant J. 2005;42(3):315-28.

5. Zhou Q, Zhu J, Cui YL, Yang ZN. Ultrastructure analysis reveals sporopollenin deposition and nexine formation at early stage of pollen wall development in Arabidopsis. Sci Bull. 2015:60(2):273-6.

6. Cecchetti V, Altamura MM, Falasca G, Costantino P, Cardarelli M. Auxin regulates Arabidopsis anther dehiscence, pollen maturation, and filament elongation. Plant Cell. 2008;20(7):1760-74.

7. Cardarelli M, Cecchetti V. Auxin polar transport in stamen formation and development: how many actors? Front Plant Sci. 2014;5:333.

8. Bennett SRM, John A, Gerd B, Smyth DR. Morphogenesis in pinoid mutants of Arabidopsis Thaliana. Plant J. 1995:8(4):505-20.

9. Benkova E, Michniewicz M, Sauer M, Teichmann T, Seifertova D, Jurgens G, Friml J. Local, efflux-dependent auxin gradients as a common module for plant organ formation. Cell. 2003;115(5):591-602

10. Cheng $Y$, Dai $X$, Zhao Y. Auxin biosynthesis by the YUCCA flavin monooxygenases controls the formation of floral organs and vascular tissues in Arabidopsis. Genes Dev. 2006:20(13):1790-9.

11. Bender RL, Fekete ML, Klinkenberg PM, Hampton M, Bauer B, Malecha M, Lindgren K, A Maki J, Perera MA, Nikolau BJ, et al. PIN6 is required for nectary auxin response and short stamen development. Plant J. 2013;74(6):893-904

12. Guilfoyle TJ, Hagen G. Auxin response factors. Curr Opin Plant Biol. 2007 10(5):453-60.

13. Guilfoyle TJ. The PB1 domain in auxin response factor and aux/IAA proteins: a versatile protein interaction module in the auxin response. Plant Cell. 2015:27(1):33-43.

14. Ellis CM, Nagpal P, Young JC, Hagen G, Guilfoyle TJ, Reed JW. Auxin response Factor 1 and Auxin response Factor2 regulate senescence and floral organ abscission in Arabidopsis Thaliana. Development. 2005; 132(20):4563-74

15. Wu MF, Tian Q, Reed JW. Arabidopsis microRNA167 controls patterns of ARF6 and ARF8 expression, and regulates both female and male reproduction. Development. 2006;133(21):4211-8.

16. Nag A, King S. Jack T: miR319a targeting of TCP4 is critical for peta growth and development in Arabidopsis. Proc Natl Acad Sci U S A. 2009;106(52):22534-9.

17. Yang J, Tian L, Sun MX, Huang XY, Zhu J, Guan YF, Jia QS, Yang ZN. Auxin response Factor17 is essential for pollen wall pattern formation in Arabidopsis. Plant Physiol. 2013:162(2):720-31.

18. Pillai RS, Bhattacharyya SN, Filipowicz W. Repression of protein synthesis by miRNAs: how many mechanisms? Trends Cell Biol. 2007;17(3):118-26.

19. Iwakawa HO, Tomari Y. Molecular insights into microRNA-mediated translational repression in plants. Mol Cell. 2013;52(4):591-601.

20. Mallory AC, Bartel DP, Bartel B. MicroRNA-directed regulation of Arabidopsis Auxin response Factor17 is essential for proper development and modulates expression of early auxin response genes. Plant Cell. 2005;17(5):1360-75.

21. Liu X, Huang J, Wang Y, Khanna K, Xie Z, Owen HA, Zhao D. The role of floral organs in carpels, an Arabidopsis loss-of-function mutation in MicroRNA160a, in organogenesis and the mechanism regulating its expression. Plant J. 2010;62(3):416-28. 
22. Feng $X$, Dickinson HG. Tapetal cell fate, lineage and proliferation in the Arabidopsis anther. Development. 2010;137(14):2409-16.

23. Xu XF, Wang B, Lou Y, Han WJ, Lu JY, Li DD, Li LG, Zhu J, Yang ZN. Magnesium transporter 5 plays an important role in $\mathrm{mg}$ transport for male gametophyte development in Arabidopsis. Plant J. 2015;84(5):925-36.

24. Mi H, Muruganujan A, Casagrande JT, Thomas PD. Large-scale gene function analysis with the PANTHER classification system. Nat Protoc. 2013; 8(8):1551-66.

25. Bruex A, Kainkaryam RM, Wieckowski Y, Kang YH, Bernhardt C, Xia Y, Zheng $X$, Wang JY, Lee MM, Benfey P, et al. A gene regulatory network for root epidermis cell differentiation in Arabidopsis. PLoS Genet. 2012;8(1):e1002446.

26. Kuroha T, Okuda A, Arai M, Komatsu Y, Sato S, Kato T, Tabata S, Satoh S. Identification of Arabidopsis subtilisin-like serine protease specifically expressed in root stele by gene trapping. Physiol Plant. 2009;137(3):281-8.

27. Kodaira KS, Qin F, Tran LS, Maruyama K, Kidokoro S, Fujita Y, Shinozaki K, Yamaguchi-Shinozaki K. Arabidopsis Cys2/His2 zinc-finger proteins AZF1 and AZF2 negatively regulate abscisic acid-repressive and auxin-inducible genes under abiotic stress conditions. Plant Physiol. 2011;157(2):742-56.

28. Zhu J, Chen H, Li H, Gao JF, Jiang H, Wang C, Guan YF, Yang ZN. Defective in Tapetal development and function 1 is essential for anther development and tapetal function for microspore maturation in Arabidopsis. Plant J. 2008; 55(2):266-77.

29. Gu JN, Zhu J, Yu Y, Teng XD, Lou Y, Xu XF, Liu JL, Yang ZN. DYT1 directly regulates the expression of TDF1 for tapetum development and pollen wall formation in Arabidopsis. Plant J. 2014;80(6):1005-13.

30. Lou Y, Xu XF, Zhu J, Gu JN, Blackmore S, Yang ZN. The tapetal AHL family protein TEK determines nexine formation in the pollen wall. Nat Commun. 2014;5:3855.

31. Xiong SX, Lu JY, Lou Y, Teng XD, Gu JN, Zhang C, Shi QS, Yang ZN, Zhu J. The transcription factors MS188 and AMS form a complex to activate the expression of CYP703A2 for sporopollenin biosynthesis in Arabidopsis Thaliana. Plant J. 2016;88(6):936-46.

32. Wang JW, Wang LJ, Mao YB, Cai WJ, Xue HW, Chen XY. Control of root cap formation by MicroRNA-targeted auxin response factors in Arabidopsis. Plant Cell. 2005;17(8):2204-16.

33. Jing Q, Huang S, Guth S, Zarubin T, Motoyama A, Chen J, Di Padova F, Lin SC, Gram H, Han J. Involvement of microRNA in AU-rich element-mediated mRNA instability. Cell. 2005;120(5):623-34.

34. Franks TM, Lykke-Andersen J. TTP and BRF proteins nucleate processing body formation to silence mRNAs with AU-rich elements. Genes Dev. 2007; 21(6):719-35.

35. Shi ZH, Zhang C, Xu XF, Zhu J, Zhou Q, Ma LJ, Niu J, Yang ZN.

Overexpression of AtTTP affects ARF17 expression and leads to male sterility in Arabidopsis. PLoS One. 2015;10(3):e0117317.

\section{Submit your next manuscript to BioMed Central and we will help you at every step:}

- We accept pre-submission inquiries

- Our selector tool helps you to find the most relevant journal

- We provide round the clock customer support

- Convenient online submission

- Thorough peer review

- Inclusion in PubMed and all major indexing services

- Maximum visibility for your research

Submit your manuscript at www.biomedcentral.com/submit
C Biomed Central 